\title{
Synthesis of Aza Coumarines by Nucleophilic Addition of Acetylenic Esters Catalysed by $\mathbf{P h}_{3} \mathbf{P}$
}

\author{
Bita Mohtat, ${ }^{\text {a,* }}$ Issa Yavari, ${ }^{\mathrm{b}}$ Roza E. Ameri, ${ }^{\mathrm{a}}$ and Elnaz Salahi ${ }^{\mathrm{a}}$ \\ ${ }^{a}$ Chemistry Department, Islamic Azad University, Karaj Branch, Karaj, Iran \\ ${ }^{\mathrm{b}}$ Chemistry Department, Islamic Azad University, Science and Research Branch, Tehran, Iran
}

RECEIVED JUNE 16, 2010; REVISED NOVEMBER 28, 2010; ACCEPTED DECEMBER 27, 2010

\begin{abstract}
Hydroxypyridine undergoes a smooth reaction with dialkyl acetylenedicarboxylates in the presence of triphenylphosphine to produce alkyl 2-oxo- $2 \mathrm{H}$-pyrano[3,2-b]pyridine-4-carboxylate and dialkyl -2-[4(alkoxycarbonyl)-2-oxo-2- $H$-pyrano[3,2-b]pyridin-6-yl]-2-butenedioate in high yields. (doi: $10.5562 /$ cca1725)
\end{abstract}

Keywords: 3-hydroxypyridine, coumarine, triphenylphosphine, acetylenic ester, coumarine

\section{INTRODUCTION}

Coumarins are of interest because they constitute an important class of naturally occurring compounds, many of which exhibit useful and diverse biological activity. ${ }^{1}$ In addition, other coumarins are of much interest a result of their toxicity, ${ }^{2}$ carcinogenicity, ${ }^{3}$ and photodynamic effects. ${ }^{4}$ In particular, those coumarins fused to pyridines have been reported to possess antiallergic, ${ }^{5}$ antidiabetic, ${ }^{6}$ and analgesic properties. ${ }^{7}$ Also, a considerable number of natural or synthetic derivatives of coumarin have found pharmaceutical applications. ${ }^{8,9}$ Thus, the synthesis of this heterocyclic nucleus is of current interest. Coumarins and aza cumarins have been synthesized by several methods. ${ }^{10-14}$ We have recently described a new and operationally convenient approach to the synthesis of carboxymethyl coumarines based on aromatic electrophilic substitution reaction between the conjugate base of substituted phenols, catechol, resorcinol, hydroquinone, and pyrogallol. ${ }^{15,16}$ In continuation of our current interest in the development of new routes to heterocyclic and carbocyclic systems, ${ }^{17-19}$ we report here a simple one-pot synthesis of functionalized 5-Aza coumarines 3,4 . Thus, reaction of 3-hydroxypyridine 1 with dialkyl acetylenedicarboxylate $\mathbf{2}$ in the presence of triphenylphosphine leads to the corresponding coumarins $\mathbf{3}, \mathbf{4}$ (Scheme 1).

\section{RESULT AND DISCUSSION}

The reaction of 2-hydroxy pyridine (1) with dialkyl acetylenedicarboxylate (2) in the presence of $\mathrm{Ph}_{3} \mathrm{P}$ proceeded spontaneously in toluene at reflux temperature and needed within $24 \mathrm{~h}$ to finish. On the other conditions (changing solvents, variations of molar ratios)

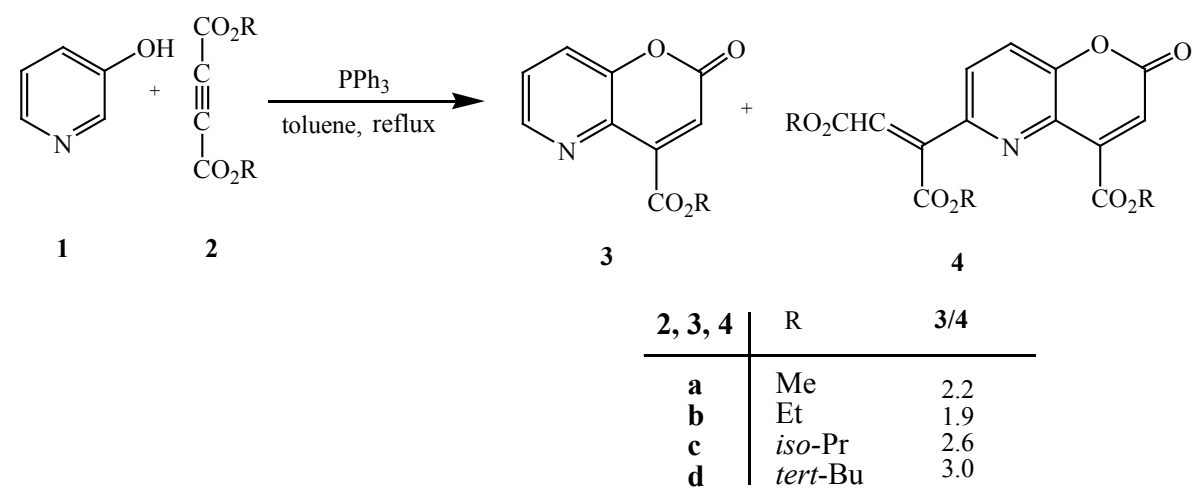

Scheme 1.

\footnotetext{
* Author to whom correspondence should be addressed. (E-mail: b.mohtat@jooyan.org)
} 


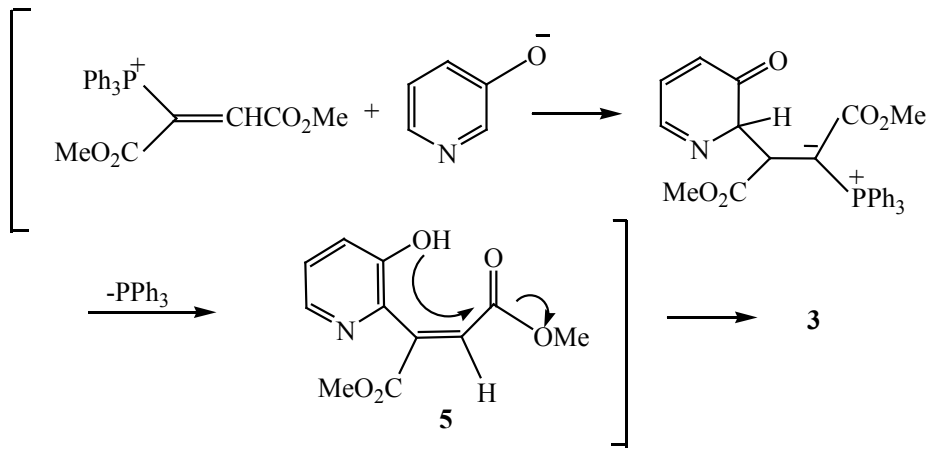

Scheme 2.

either did not have any considerable effect on $\mathbf{3}$ and $\mathbf{4}$ yields or it caused reaction failed to proceed. The products were separated by column chromatography and identified as $\mathbf{3}$ and $\mathbf{4}$ based on their elemental analyses and their IR, ${ }^{1} \mathrm{H}$, and ${ }^{13} \mathrm{C}$ NMR spectral data. The mass spectra of these compounds displayed molecular ion peaks at appropriate $\mathrm{m} / \mathrm{z}$ values. The ${ }^{1} \mathrm{H}$ NMR spectrum of $\mathbf{3 a}$ exhibited a single sharp line for the methyl group at $\delta=4.04 \mathrm{ppm}$, together with singlet at $\delta=6.84 \mathrm{ppm}$ for methine proton. The aromatic moiety was appeared at $7.54-8.66 \mathrm{ppm}$. The ${ }^{13} \mathrm{C}$ NMR spectrum of 3a showed ten distinct resonances in agreement with the methyl 2-oxo- $2 H$-pyrano[3,2b]pyridine-4-carboxylate structure. The ${ }^{1} \mathrm{H}$ and ${ }^{13} \mathrm{C}$ NMR spectra of $\mathbf{3 b}$ and $\mathbf{3} \mathbf{c}$ are similar to those of $\mathbf{3 a}$ except for the alkoxy moieties, which exhibited characteristic resonances with appropriate chemical shifts. The ${ }^{1} \mathrm{H}$ NMR spectrum of $\mathbf{4 a}$ exhibited three single sharp line for the methoxy group at $\delta=3.84,3.99$ and $4.05 \mathrm{ppm}$, together with two singlet at $\delta=6.88$ and $6.89 \mathrm{ppm}$ for methine protons. The ${ }^{13} \mathrm{C}$ NMR spectrum of 4a exhibited fifteen sharp lines in agreement with the proposed structure. Partial assignment of these resonances is given in the experimental.

Mechanistically, ${ }^{15,16}$ it is conceivable that the reaction involves the initial formation of a zwitterionic intermediate between $\mathrm{Ph}_{3} \mathrm{P}$ and the acetylenic compound and subsequent protonation of reactive 1:1 adduct followed by electrophilic attack of vinyltriphenylphosphonium cation on the aromatic ring at the ortho position relative to the strong activation group. The product is presumably produced by intramolecular lactonization of the unsaturated diester $\mathbf{5}$ (Scheme 2).

Reaction leading to $\mathbf{4}$, involves formation of the zwitterionic intermediate which is protonated by 3hydroxypyridine. Then it is attacked by the conjugate base to produce ylide, in next step the intermediate undergoes proton transfer to furnish the 1,3-diionic structure. At the end, it is converted to the final product by loss of $\mathrm{Ph}_{3} \mathrm{P}$.

\section{CONCLUSION}

In conclusion, we have described a convenient route to aza coumarines, through nucleophilic addition to dialkyl acetylenedicarboxylate. These functionalised coumarines may be considered as potentially useful synthetic intermediates because they possess atoms with different oxidation states. The present method has the advantages that not only are the reaction performed under neutral conditions, but also the substances can be mixed without any modification. The simplicity of the present procedure makes it an interesting alternative to other approaches.

\section{EXPERIMENTAL}

\section{General procedure}

Compounds 1, 2 and $\mathrm{Ph}_{3} \mathrm{P}$ were obtained from Fluka and were used without further purification. M.p. Electrothermal-9100 apparatus. IR Spectra: Shimadzu IR460 spectrometer. ${ }^{1} \mathrm{H}$ and ${ }^{13} \mathrm{C}$ NMR spectra: Bruker DRX-500 AVANCE instrument; in $\mathrm{CDCl}_{3}$ at 300 and $75 \mathrm{MHz}$, respectively; $\delta$ in ppm. EI-MS $(70 \mathrm{eV})$ : Finnigan-MAT-8430 mass spectrometer, in $\mathrm{m} / \mathrm{z}$. Elemental analyses $(\mathrm{C}, \mathrm{H}, \mathrm{N})$ were performed with a Heraeus CHN-O-Rapid analyser.

General Procedure for the Preparation of compound 3 To a stirred solution of $0.52 \mathrm{~g}$ of $\mathrm{Ph}_{3} \mathrm{P}(2 \mathrm{mmol})$ and $0.19 \mathrm{~g} 1(2 \mathrm{mmol})$ in toluene $(10 \mathrm{ml})$ was added drop wise a mixture of $2(2 \mathrm{mmol})$ in toluene $(2 \mathrm{ml})$ at room temperature over $5 \mathrm{~min}$. The reaction mixture was heated under reflux for $24 \mathrm{~h}$. The solvent was removed under reduced pressure, and the viscous residue was purified by column chromatography ( $\mathrm{SiO} 2$; hexane/AcOEt 4:1) to afford the pure adducts.

Methyl 2-oxo-2H-pyrano[3,2-b]pyridine-4-carboxylate (3a) Yellow powder, $0.20 \mathrm{~g}(49 \%)$; mp $128-132{ }^{\circ} \mathrm{C}$; IR $(\mathrm{KBr}) v_{\max } / \mathrm{cm}^{-1}: 1744(\mathrm{C}=\mathrm{O}), 1625(\mathrm{C}=\mathrm{N}) ;{ }^{1} \mathrm{H}$ NMR $\left(\mathrm{CDCl}_{3}\right) \delta / \mathrm{ppm}: 4.04$ (s, 3H, Me), $6.84(\mathrm{~s}, 1 \mathrm{H}, \mathrm{CH}), 7.54$ 
(dd, 1H, $J=7.1,4.5 \mathrm{~Hz}, \mathrm{CH}) 7.69$ (d, 1H, $J=7.1, \mathrm{CH}$ ), $8.66(\mathrm{~d}, 1 \mathrm{H}, J=4.5 \mathrm{~Hz}, \mathrm{CH}) .{ }^{13} \mathrm{C}$ NMR $\left(\mathrm{CDCl}_{3}\right) \delta / \mathrm{ppm}$ : $53.8(\mathrm{Me}), 120.1,125.1,126.9(\mathrm{CH}), 134.9,146.8(\mathrm{C})$, $147.3(\mathrm{CH}), 151.3(\mathrm{C}), 159.0,164.3(\mathrm{C}=\mathrm{O}) . \mathrm{MS}(\mathrm{EI})$ $\mathrm{m} / \mathrm{z}: 205\left(\mathrm{M}^{+}, 47 \%\right), 190\left(\left[\mathrm{M}-\mathrm{CH}_{3}\right]^{+}, 68 \%\right), 161([\mathrm{M}-$ $\left.\left.\mathrm{CO}_{2}\right]^{+}, 100\right), 146\left(\left[\mathrm{M}-\mathrm{CO}_{2}-\mathrm{CH}_{3}\right]^{+}, 45 \%\right)$.

Anal. Calcd. mass fraction of elements, $w / \%$ for $\mathrm{C}_{10} \mathrm{H}_{7} \mathrm{NO}_{4}\left(M_{\mathrm{r}}=205.17\right)$ are: $\mathrm{C} 58.54, \mathrm{H} 3.44, \mathrm{~N} 6.83$; Found: C 57.93, H 3.56, N 6.88.

Dimethyl-2-[4-(methoxycarbonyl)-2-oxo-2-H-pyrano[3,2-b]pyridin-6-yl]-2-butenedioate (4a)

Yellow oil, $0.26 \mathrm{~g}(37 \%)$; IR (KBr) $v_{\max } / \mathrm{cm}^{-1}: 1744$ $(\mathrm{C}=\mathrm{O}), 1636(\mathrm{C}=\mathrm{N}) ;{ }^{1} \mathrm{H} \mathrm{NMR}\left(\mathrm{CDCl}_{3}\right) \delta / \mathrm{ppm}: 3.84(\mathrm{~s}$, $3 \mathrm{H}, \mathrm{Me}), 3.99$ (s, 3H, Me), 4.05 (s, 3H, Me), 6.88 (s, $1 \mathrm{H}, \mathrm{CH}), 6.89$ (s, 1H, CH), 7.70 (brs, 2H, $2 \mathrm{CH}) .{ }^{13} \mathrm{C}$ NMR $\left(\mathrm{CDCl}_{3}\right) \delta / \mathrm{ppm}: 52.8,53.3,53.8(\mathrm{Me}), 120.9$, 121.4, 124.9, $125.9(\mathrm{CH}), 146.1,146.6,148.3,151.2$, 158.3 (C), 163.7, 165.4, $167.6(\mathrm{C}=\mathrm{O})$; MS (EI) $\mathrm{m} / \mathrm{z}: 347$ $\left(\mathrm{M}^{+}, 37 \%\right), 303\left(\left[\mathrm{M}-\mathrm{CO}_{2}\right]^{+}, 84 \%\right), 292\left(\left[\mathrm{M}-3 \mathrm{CH}_{3}\right]^{+}\right.$, $100 \%), 288\left(\left[\mathrm{M}-\mathrm{CO}_{2}-\mathrm{CH}_{3}\right]^{+}, 15 \%\right), 204\left(\left[\mathrm{M}-\mathrm{C}_{6} \mathrm{H}_{7} \mathrm{O}_{4}\right]^{+}\right.$, $39 \%), 170\left(\left[\mathrm{M}-3 \mathrm{CO}_{2}-\mathrm{CH}_{3}\right]^{+}, 100 \%\right)$.

Anal. Calcd. mass fraction of elements, $w / \%$ for $\mathrm{C}_{16} \mathrm{H}_{13} \mathrm{NO}_{8}\left(M_{\mathrm{r}}=347.28\right)$ are: $\mathrm{C} 55.34, \mathrm{H} 3.77, \mathrm{~N} 4.03$; Found: C 55.96, H 3.09, N 4.88.

\section{Ethyl-2-oxo-2H-pyrano[3,2-b]pyridine-4-carboxylate}

(3b)

Yellow powder, $0.18 \mathrm{~g}(41 \%) ; \mathrm{mp} 119-121{ }^{\circ} \mathrm{C} . \mathrm{IR}$ $(\mathrm{KBr}) v_{\max } / \mathrm{cm}^{-1}: 1740(\mathrm{C}=\mathrm{O}), 1634(\mathrm{C}=\mathrm{N}) ;{ }^{1} \mathrm{H}$ NMR $\left(\mathrm{CDCl}_{3}\right) \delta / \mathrm{ppm}: 1.44$ (t, 3H, $\left.J=7.1 \mathrm{~Hz}, \mathrm{Me}\right), 4.52$ (q, $\left.2 \mathrm{H}, J=7.1 \mathrm{~Hz}, \mathrm{OCH}_{2}\right), 6.83(\mathrm{~s}, 1 \mathrm{H}, \mathrm{CH}), 7.53(\mathrm{dd}, 1 \mathrm{H}$, $J=7.1,4.5 \mathrm{~Hz}, \mathrm{CH}), 7.69(\mathrm{~d}, 1 \mathrm{H}, J=7.1, \mathrm{~Hz}, \mathrm{CH}), 8.66$ $(\mathrm{d}, 1 \mathrm{H}, J=4.5 \mathrm{~Hz}, \mathrm{CH}) .{ }^{13} \mathrm{C} \mathrm{NMR}\left(\mathrm{CDCl}_{3}\right) \delta / \mathrm{ppm}: 14.5$ (Me), $63.2\left(\mathrm{OCH}_{2}\right), 119.7,125.1,126.8(\mathrm{CH}), 139.3$, $147.1(\mathrm{C}), 147.3(\mathrm{CH}), 151.3(\mathrm{C}), 159.1,163.8(\mathrm{C}=\mathrm{O})$. MS (EI) $m / z: 219\left(\mathrm{M}^{+}, 18 \%\right), 190\left(\left[\mathrm{M}-\mathrm{CH}_{3}\right]^{+}, 51 \%\right)$, $175\left(\left[\mathrm{M}-\mathrm{CO}_{2}\right]^{+}, 90 \%\right), 146\left(\left[\mathrm{M}-\mathrm{CO}_{2}-\mathrm{C}_{2} \mathrm{H}_{5}\right]^{+}, 68 \%\right)$.

Anal. Calcd. mass fraction of elements, $w / \%$ for $\mathrm{C}_{11} \mathrm{H}_{9} \mathrm{NO}_{4}\left(M_{\mathrm{r}}=219.19\right)$ are: $\mathrm{C} 60.28, \mathrm{H} 4.14, \mathrm{~N} 6.39$; Found: C 60.32, H 3.78, N 6.36 .

Diethyl-2-[4-(ethoxycarbonyl)-2-oxo-2-H- pyrano[3,2b]pyridin-6-yl]-2-butenedioate (4b)

Yellow oil, $0.30 \mathrm{~g}(39 \%)$. IR (KBr) $v_{\max } / \mathrm{cm}^{-1}: 1728$ $(\mathrm{C}=\mathrm{O}), 1624(\mathrm{C}=\mathrm{N}) ;{ }^{1} \mathrm{H}$ NMR $\left(\mathrm{CDCl}_{3}\right) \delta / \mathrm{ppm}: 1.35(\mathrm{t}$, $3 \mathrm{H}, J=7.0 \mathrm{~Hz}, \mathrm{Me}$ ), 1.41 (t, $3 \mathrm{H}, J=7.0 \mathrm{~Hz}, \mathrm{Me}), 1.47$ (t, 3H, $J=7.1 \mathrm{~Hz}, \mathrm{Me}$ ), 4.29 (q, 2H, $J=7.1 \mathrm{~Hz}, \mathrm{OCH}_{2}$ ), 4.46 (q, 2H, $\left.J=7.1 \mathrm{~Hz}, \mathrm{OCH}_{2}\right), 4.52(\mathrm{q}, 2 \mathrm{H}, J=7.1 \mathrm{~Hz}$, $\left.\mathrm{OCH}_{2}\right), 6.87$ (s, 1H, CH), 6.92 (s, 1H, CH), 7.69 (brs, $2 \mathrm{H}, 2 \mathrm{CH}) .{ }^{13} \mathrm{C} \mathrm{NMR}\left(\mathrm{CDCl}_{3}\right) \delta / \mathrm{ppm}: 14.4,14.5,14.6$ (Me), 61.7, 62.5, $63.2\left(\mathrm{OCH}_{2}\right), 120.5,122.1,125.5$, $125.8(\mathrm{CH}), 145.5,147.1,148.5,151.2,158.5(\mathrm{C})$, 163.5, 165.0, $167.1(\mathrm{C}=\mathrm{O})$. MS (EI) $m / z: 389\left(\mathrm{M}^{+}, 26\right.$ $\%), 345\left(\left[\mathrm{M}-\mathrm{CO}_{2}\right]^{+}, 77 \%\right), 316\left(\left[\mathrm{M}-\mathrm{CO}_{2}-\mathrm{C}_{2} \mathrm{H}_{5}\right]^{+}, 48\right.$ $\%), 302\left(\left[\mathrm{M}-3 \mathrm{C}_{2} \mathrm{H}_{5}\right]^{+}, 59 \%\right), 218\left(\left[\mathrm{M}-\mathrm{C}_{8} \mathrm{H}_{11} \mathrm{O}_{4}\right]^{+}, 76\right.$
$\%), 170\left(\left[\mathrm{M}-3 \mathrm{CO}_{2}-\mathrm{C}_{2} \mathrm{H}_{5}\right]^{+}, 100 \%\right)$.

Anal. Calcd. mass fraction of elements, $w / \%$ for $\mathrm{C}_{19} \mathrm{H}_{19} \mathrm{NO}_{8}\left(M_{\mathrm{r}}=389.36\right)$ are: $\mathrm{C} 58.61, \mathrm{H} 4.92, \mathrm{~N} 3.60$; Found: C 58.20, H 4.68, N 5.15.

Isopropyl-2-oxo-2H-pyrano[3,2-b]pyridine-4-carboxylate (3c)

Yellow powder, $0.22 \mathrm{~g}(48 \%) . \mathrm{mp} 125-128{ }^{\circ} \mathrm{C}$. IR $(\mathrm{KBr}) v_{\max } / \mathrm{cm}^{-1}: 1742(\mathrm{C}=\mathrm{O}), 1622(\mathrm{C}=\mathrm{N}) ;{ }^{1} \mathrm{H}$ NMR $\left(\mathrm{CDCl}_{3}\right) \delta / \mathrm{ppm}: 1.43(\mathrm{~d}, 6 \mathrm{H}, J=6.0 \mathrm{~Hz}, 2 \mathrm{Me}), 5.41$ (sept, $1 \mathrm{H}, J=6.2 \mathrm{~Hz}, \mathrm{OCH}), 7.52(\mathrm{dd}, 1 \mathrm{H}, J=7.1,4.5$ $\mathrm{Hz}, \mathrm{CH}), 7.67$ (d, 1H, $J=7.1 \mathrm{~Hz}, \mathrm{CH}), 8.66(\mathrm{~d}, 1 \mathrm{H}, J=$ $4.5 \mathrm{~Hz}, \mathrm{CH}) .{ }^{13} \mathrm{C}$ NMR $\left(\mathrm{CDCl}_{3}\right) \delta / \mathrm{ppm}: 22.2$ (2Me), 71.2, 119.3, 124.9, $126.7(\mathrm{CH}), 135.5(\mathrm{C}), 147.3(\mathrm{CH})$, 147.5, $151.3(\mathrm{C}), 159.1,163.3(\mathrm{C}=\mathrm{O})$. MS (EI) $\mathrm{m} / \mathrm{z}: 233$ $\left(\mathrm{M}^{+}, 12 \%\right), 190\left(\left[\mathrm{M}-\mathrm{C}_{3} \mathrm{H}_{7}\right]^{+}, 35 \%\right), 189\left(\left[\mathrm{M}-\mathrm{CO}_{2}\right]^{+}, 58\right.$ $\%), 146\left(\left[\mathrm{M}-\mathrm{CO}_{2}-\mathrm{C}_{3} \mathrm{H}_{7}\right]^{+}, 100 \%\right)$.

Anal. Calcd. mass fraction of elements, $w / \%$ for $\mathrm{C}_{12} \mathrm{H}_{11} \mathrm{NO}_{4}\left(M_{\mathrm{r}}=233.22\right)$ are: $\mathrm{C} 61.80, \mathrm{H} 4.75, \mathrm{~N} 6.01$; Found: C 61.33, H 5.06, N 6.42 .

Diisopropylyl-2-[4-(isopropoxycarbonyl)-2-oxo-2-Hpyrano[3,2-b]pyridin-6-yl]-2-butenedioate (4c)

Yellow oil, $0.29 \mathrm{~g}(34 \%)$. IR (KBr) $v_{\max } / \mathrm{cm}^{-1}: 1743$ $(\mathrm{C}=\mathrm{O}), 1634(\mathrm{C}=\mathrm{N}) ;{ }^{1} \mathrm{H}$ NMR $\left(\mathrm{CDCl}_{3}\right) \delta / \mathrm{ppm}: 1.31(\mathrm{~d}$, $6 \mathrm{H}, J=6.2 \mathrm{~Hz}, 2 \mathrm{Me}), 1.40(\mathrm{~d}, 6 \mathrm{H}, J=6.3 \mathrm{~Hz}, 2 \mathrm{Me})$, $1.45(\mathrm{~d}, 6 \mathrm{H}, J=6.3 \mathrm{~Hz}, 6 \mathrm{H}, 2 \mathrm{Me}), 5.12$ (sept, $1 \mathrm{H}, J=$ $6.3 \mathrm{~Hz}, 1 \mathrm{H}, \mathrm{OCH}), 5.36$ (sept, $1 \mathrm{H}, J=6.2 \mathrm{~Hz}, \mathrm{OCH}$ ), 5.38 (sept, $1 \mathrm{H}, J=6.3 \mathrm{~Hz}, \mathrm{OCH}), 6.83(\mathrm{~s}, 1 \mathrm{H}, \mathrm{CH})$, $6.93(\mathrm{~s}, 1 \mathrm{H}, \mathrm{CH}), 7.62(\mathrm{~d}, 1 \mathrm{H}, J=8.7 \mathrm{~Hz}, \mathrm{CH}), 7.68(\mathrm{~d}$, $1 \mathrm{H}, J=8.7 \mathrm{~Hz}, \mathrm{CH}) .{ }^{13} \mathrm{C} \mathrm{NMR}\left(\mathrm{CDCl}_{3}\right) \delta / \mathrm{ppm}: 22.0$, 22.2 (2Me), 69.2, 70.3, 71.4, 120.2, 122.7, 124.9, 125.7 (CH), 145.4, 147.6, 149.0, 151.2, 158.5 (C), 163.2, 164.4, $166.6(\mathrm{C}=\mathrm{O})$. MS (EI) $m / z: 431\left(\mathrm{M}^{+}, 34 \%\right), 387$ $\left(\left[\mathrm{M}-\mathrm{CO}_{2}\right]^{+}, 15 \%\right), 344\left(\left[\mathrm{M}-\mathrm{CO}_{2}-\mathrm{C}_{3} \mathrm{H}_{7}\right]^{+}, 62 \%\right), 302$ $\left(\left[\mathrm{M}-3 \mathrm{C}_{2} \mathrm{H}_{5}\right]^{+}, 100 \%\right), 232\left(\left[\mathrm{M}-\mathrm{C}_{10} \mathrm{H}_{15} \mathrm{O}_{4}\right]^{+}, 31 \%\right), 170$ $\left(\left[\mathrm{M}-3 \mathrm{CO}_{2}-\mathrm{C}_{2} \mathrm{H}_{5}\right]^{+}, 57 \%\right)$.

Anal. Calcd. mass fraction of elements, $w / \%$ for $\mathrm{C}_{22} \mathrm{H}_{25} \mathrm{NO}_{8}\left(M_{\mathrm{r}}=431.44\right)$ are: $\mathrm{C} 61.25, \mathrm{H} 5.84, \mathrm{~N} 3.25$. Found: C 61.65, H 5.36, N 3.70.

Tert-butyl 2-oxo-2H-pyrano[3,2-b]pyridine-4-carboxylate (3d)

Yellow powder, $0.19 \mathrm{~g}(38 \%)$. mp $130-132{ }^{\circ} \mathrm{C}$. IR $(\mathrm{KBr}) v_{\max } / \mathrm{cm}^{-1}: 1723(\mathrm{C}=\mathrm{O}), 1628(\mathrm{C}=\mathrm{N}) ;{ }^{1} \mathrm{H}$ NMR $\left(\mathrm{CDCl}_{3}\right) \delta / \mathrm{ppm}: 1.52(\mathrm{~s}, 9 \mathrm{H}, 3 \mathrm{Me}), 6.74(\mathrm{~s}, 1 \mathrm{H}, \mathrm{CH})$, $7.52(\mathrm{dd}, 1 \mathrm{H}, J=7.1,3.1 \mathrm{~Hz}, \mathrm{CH}), 7.67$ (d, 1H, $J=7.1$, $\mathrm{Hz}, \mathrm{CH}), 8.66(\mathrm{~d}, 1 \mathrm{H}, J=3.1 \mathrm{~Hz}, \mathrm{CH}) .{ }^{13} \mathrm{C} \mathrm{NMR}$ $\left(\mathrm{CDCl}_{3}\right) \delta /$ ppm: 28.4 (3Me), 83.6, 117.3, 118.8 (C), $124.1(\mathrm{CH}), 124.8(\mathrm{C}), 126.6(\mathrm{CH}), 143.2,147.3(\mathrm{C})$, 168.6, $1648.9(\mathrm{C}=\mathrm{O})$. MS (EI) $m / z: 247\left(\mathrm{M}^{+}, 31 \%\right), 203$ $\left(\left[\mathrm{M}-\mathrm{CO}_{2}\right]^{+}, 21 \%\right), 190\left(\left[\mathrm{M}-\mathrm{C}_{4} \mathrm{H}_{9}\right]^{+}, 100 \%\right), 146([\mathrm{M}-$ $\left.\mathrm{CO}_{2}-\mathrm{C}_{4} \mathrm{H}_{9}\right]^{+}, 81 \%$ ).

Anal. Calcd. mass fraction of elements, $w / \%$ for $\mathrm{C}_{13} \mathrm{H}_{13} \mathrm{NO}_{4}\left(M_{\mathrm{r}}=247.25\right)$ are: $\mathrm{C} 63.15, \mathrm{H} 5.30, \mathrm{~N} 5.67$. Found: C 60.89, H 5.37, N 5.73. 
Di tert-butyl-2-[4-(tert-butoxycarbonyl)-2-oxo-2-H-pyrano[3,2-b]pyridin-6-yl]-2-butenedioate (4d)

Yellow oil, $0.23 \mathrm{~g}(24 \%)$. IR (KBr) $v_{\max } / \mathrm{cm}^{-1}$ : 1723(C=O), $1633(\mathrm{C}=\mathrm{N}) ;{ }^{1} \mathrm{H}$ NMR $\left(\mathrm{CDCl}_{3}\right) \delta / \mathrm{ppm}$ : $1.52(\mathrm{~s}, 9 \mathrm{H}, 3 \mathrm{Me}), 1.62(\mathrm{~s}, 9 \mathrm{H}, 3 \mathrm{Me}), 1.67$ (s, 9H, 3Me), 6.75 (s, 1H, CH), 7.00 (s, 1H, CH), 7.59 (d, 1H, J = 7.5 $\mathrm{Hz}, \mathrm{CH}), 7.64(\mathrm{~d}, 1 \mathrm{H}, J=8.7 \mathrm{~Hz}, \mathrm{CH}) .{ }^{13} \mathrm{C}$ NMR $\left(\mathrm{CDCl}_{3}\right) \delta$ ppm: 28.4, 28.5, 28.6 (3Me), 81.7, 83.7, 85.3 (C), 119.1, 124.0, 125.0, $125.5(\mathrm{CH}), 144.6,148.4$, 148.7, 151.1, 158.9 (C), 163.0, 164.3, $166.3(\mathrm{C}=\mathrm{O})$. MS (EI) $m / z: 473\left(\mathrm{M}^{+}, 12 \%\right), 429\left(\left[\mathrm{M}-\mathrm{CO}_{2}\right]^{+}, 44 \%\right), 372$ $\left(\left[\mathrm{M}-\mathrm{CO}_{2}-\mathrm{C}_{4} \mathrm{H}_{9}\right]^{+}, 24 \%\right), 302\left(\left[\mathrm{M}-3 \mathrm{C}_{4} \mathrm{H}_{9}\right]^{+}, 89 \%\right), 246$ $\left(\left[\mathrm{M}-\mathrm{C}_{12} \mathrm{H}_{19} \mathrm{O}_{4}\right]^{+}, 58 \%\right), 170\left(\left[\mathrm{M}-3 \mathrm{CO}_{2}-\mathrm{C}_{4} \mathrm{H}_{9}\right]^{+}, 100 \%\right)$. Anal. Calcd. mass fraction of elements, $w / \%$ for $\mathrm{C}_{25} \mathrm{H}_{31} \mathrm{NO}_{8}\left(M_{\mathrm{r}}=473.52\right)$ are: $\mathrm{C} 63.41, \mathrm{H} 6.60, \mathrm{~N} 2.96$. Found: C 63.97, H 6.31, N 3.12.

\section{REFERENCES}

1. D. H. Murray, J. Mendes, and S. A. Brown, The Natural Coumarins: Occurrence, Chemistry and Biochemistry, Wiley, New York, 1982, pp. 45-46.

2. S. Kadis, A. Ciegler, and S. Ajl, Microb. Toxins 7 (1972) 497-515.

3. G. P. Ellis and G. B. West, Prog. Med. Chem. 10 (1974) 109-115.
4. P. Wells and H. Morrison, J. Am. Chem. Soc. 97 (1975) 154-159.

5. K. Ukawa, T. Ishiguro, Y. Wada, and A. Nohara, Heterocycles 24 (1986) 1931-1941.

6. D. Heber, Arch. Pharm. 320 (1987) 402-406.

7. D. Heber and T. Berghaus, J. Heterocycl. Chem. 31 (1994) 1353-1359.

8. U. P. Masche, K. M. Rentsch, A. von Felten, P. J. Meier, and K. E. Fattinger, Eur. J. Clin. Pharmacol. 54 (1999) 865-868.

9. J. Parrish, T. Fitzpatrick, L. Tannenbaum, and M. Patak, New Eng. J. Med. 291 (1974) 1207-1212.

10. D. Billeret, D. Blondeau, and H. Sliwa, J. Heterocycl. Chem. 30 (1993) 671-674.

11. G. Queguiner, F. Trecourt, F. Marsais, and T. Gungor, J. Chem. Soc. Perkin Trans 1 (1990) 2409-2415.

12. E. Galariniotou, V. Fragos, A. Makri, K. E. Litinas, and D. N Nicolaides, Tetrahedron, 63, (2007), 8298-8304.

13. F. Požgan, K. Kranjc, V. Kepe, S. Polanc, and M. Kočevar, Arkivoc (viii) (2007) 67-111.

14. A. Goel and V. J. Ram, Tetrahedron 65 (2009) 7865-7913.

15. I. Yavari, R. Hekmat-Shoar, and A. Zonouzi, Tetrahedron Lett. 39 (1998) 2391-2392.

16. I. Yavari, M. Adib, and L. Hojabri, Tetrahedron 58 (2002) 6895-6599.

17. I. Yavari, B. Mohtat, and H. Zare, Mendeleev Commun 2 (2006) 102-104.

18. I. Yavari, A. R. Alborzi, B. Mohtat, and F. Nourmohammadian, Synthetic Communications 38 (2008) 1-10.

19. B. Mohtat, S. Jabbar, A. Ghasemi, and I. Yavari, J. Chem. Res (2008) 601-603. 